\title{
ON THE SYMMETRY OF MATRIX ALGEBRAS
}

\author{
JOSEF WICHMANN
}

\begin{abstract}
A *-algebra is called symmetric, if each element of the form $a^{*} a$ has nonnegative real spectrum. The study of locally compact groups with symmetric group algebras led to the following theorem: The tensoring of a Banach *-algebra with the *-algebra of all complex $n \times n$ matrices preserves symmetry. In this note we prove, by a very simple algebraic argument, an analogue of it for arbitrary ${ }^{*}$-algebras.
\end{abstract}

1. Introduction. The problem of determining which locally compact groups have symmetric group algebras has received much attention. It of ten led to the study of the question whether the tensor product of two symmetric Banach * algebras $A$ and $B$ is again symmetric [2], [1], [4], [5].

In the special case where $B$ is the finite dimensional ${ }^{*}$-algebra $C_{n}$ of all complex $n \times n$ matrices the tensor product of $A$ and $B$ is the ${ }^{*}$-algebra $A_{n}$ of all $n \times n$ matrices over $A$ with the usual algebraic operations and involution $\left(a_{i j}\right)^{*}=\left(a_{j i}^{*}\right)$. It was proved by H. Leptin [6] and the author [7] that if $A$ is a symmetric Banach $*^{*}$-algebra, then the matrix algebra $A_{n}$ is also symmetric. (As pointed out in [6] D. W. Bailey's proof [1] of the $2 \times 2$ case is incorrect.)

The purpose of this note is to prove, by a very simple algebraic argument, the following more general result.

TheOREM. Let $A$ be $a{ }^{*}$-algebra and let $n$ be a positive integer. Then $A$ has the property that each element of the form $a_{1}^{*} a_{1}+\cdots+a_{k}^{*} a_{k}, k=1,2,3, \ldots$, has nonnegative real spectrum if and only if the ${ }^{*}$-algebra $A_{n}$ of all $n \times n$ matrices over $A$ has the same property.

This answers a question of R. S. Doran [3].

2. Proof of the Theorem. Let $A$ be an arbitrary *-algebra and let $k$ and $n$ be positive integers. For convenience we say that $A$ is $k$-symmetric, if for every set of $k$ elements $a_{1}, \ldots, a_{k}$ in $A$ the element $a_{1}^{*} a_{1}+\cdots+a_{k}^{*} a_{k}$ has

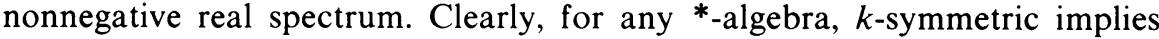
symmetric, i.e. 1 -symmetric. It is well known that for Banach *-algebras the converse holds.

2.1. Lemma. If $A$ is $k$-symmetric, then the *-algebra $A_{e}$ obtained from $A$ by adjunction of an identity element $e$ is also $k$-symmetric.

If the *-algebra $\left(A_{e}\right)_{n}$ of all $n \times n$ matrices over $A_{e}$ is $k$-symmetric, then the *algebra $A_{n}$ of all $n \times n$ matrices over $A$ is also k-symmetric.

Proof. Given a $k$-tuple $\lambda=\left(\lambda_{1}, \ldots, \lambda_{k}\right)$ of complex scalars and a set of $k$

Received by the editors February 21, 1975 and, in revised form, April 22, 1975.

AMS (MOS) subject classifications (1970). Primary 46K99, 43A20.

Key words and phrases. Matrix algebra, ${ }^{*}$-algebra, group algebra, symmetry. 
elements $a_{1}, \ldots, a_{k}$ in $A$, set

$$
h=\sum\left(\lambda_{r} e+a_{r}\right)^{*}\left(\lambda_{r} e+a_{r}\right)-\sum\left|\lambda_{r}\right|^{2} e \text { and }|\lambda|^{2}=\sum\left|\lambda_{r}\right|^{2}
$$

(sums are taken over $r=1, \ldots, k$ ). Since $h$ is a Hermitian element in $A$ we can decompose the following element into commuting factors.

$$
\begin{aligned}
e+\sum & {\left[\left(\lambda_{r} e+a_{r}\right)\left(1+|\lambda|^{2}\right)^{-1} h\right]^{*}\left[\left(\lambda_{r} e+a_{r}\right)\left(1+|\lambda|^{2}\right)^{-1} h\right] } \\
& =e+\sum h\left(1+|\lambda|^{2}\right)^{-1}\left(\lambda_{r} e+a_{r}\right)^{*}\left(\lambda_{r} e+a_{r}\right)\left(1+|\lambda|^{2}\right)^{-1} h \\
& =e+h\left(1+|\lambda|^{2}\right)^{-1}\left(h+|\lambda|^{2} e\right)\left(1+|\lambda|^{2}\right)^{-1} h \\
& =\left(e+h\left(1+|\lambda|^{2}\right)^{-1}(h-e)\right)\left(1+|\lambda|^{2}\right)^{-1}\left(e+|\lambda|^{2} e+h\right) .
\end{aligned}
$$

Thus, if $A$ is $k$-symmetric, then

$$
e+|\lambda|^{2} e+h=e+\sum\left(\lambda_{r} e+a_{r}\right)^{*}\left(\lambda_{r} e+a_{r}\right)
$$

is invertible in $A_{e}$, i.e. $A_{e}$ is $k$-symmetric (see also [7]).

The assertion about $A_{n}$ follows from the fact that $A_{n}$ is a ${ }^{*}$-ideal in $\left(A_{e}\right)_{n}$.

The preceding lemma shows that we may assume without loss of generality that $A$ has an identity element $e$. We denote the identity matrix in $A_{n}$ by $e_{n}$.

2.2. REMARK. If $A_{n}$ is $k$-symmetric, then $A$ is $(n \cdot k)$-symmetric. Indeed, if $A_{n}$ is $k$-symmetric, then for the $n \times n$ matrices $a_{r}=\left(a_{r i j}\right)$, with $a_{r i j}=0$ for $j>1$, the $n \times n$ matrix

$$
e_{n}+\sum a_{r}^{*} a_{r}=\left(\begin{array}{cc}
e+\sum\left(a_{r 11}^{*} a_{r 11}+\cdots+a_{r n 1}^{*} a_{r n 1}\right) & 0 \\
0 & e_{n-1}
\end{array}\right)
$$

is invertible in $A_{n}$. Thus $e+\sum\left(a_{r 11}^{*} a_{r 11}+\cdots+a_{r n 1}^{*} a_{r n 1}\right)$ is invertible in $A$, i.e. $A$ is $(n \cdot k)$-symmetric. This proves one direction of the theorem.

2.3. LemMA. If $A$ is $(2 k+1)$-symmetric, then the *algebra $A_{2}$ is $k$-symmetric.

Proof. Given $k$ matrices $a_{r}=\left(a_{r i j}\right), r=1, \ldots, k$, in $A_{2}$, we have to show that the matrix

$$
\begin{aligned}
& e_{2}+\sum a_{r}^{*} a_{r} \\
& \quad=\left(\begin{array}{cc}
e+\sum\left(a_{r 11}^{*} a_{r 11}+a_{r 21}^{*} a_{r 21}\right) & b \\
b^{*} & e+\sum\left(a_{r 12}^{*} a_{r 12}+a_{r 22}^{*} a_{r 22}\right)
\end{array}\right)
\end{aligned}
$$

is invertible in $A_{2}$. Consider the invertible $2 \times 2$ matrix

$$
x=\left(\begin{array}{cc}
e & -\left(e+\sum\left(a_{r 11}^{*} a_{r 11}+a_{r 21}^{*} a_{r 21}\right)\right)^{-1} b \\
0 & e
\end{array}\right),
$$

and set $y_{r}=a_{r} x$. Simple matrix multiplication shows that

$$
\begin{aligned}
x^{*}\left(e_{2}+\sum a_{r}^{*} a_{r}\right) x=x^{*} x+\sum y_{r}^{*} y_{r} & \\
\quad= & \left(\begin{array}{cc}
e+\sum\left(a_{r 11}^{*} a_{r 11}+a_{r 21}^{*} a_{r 21}\right) & 0 \\
0 & e+x_{12}^{*} x_{12}+\sum\left(y_{r 12}^{*} y_{r 12}+y_{r 22}^{*} y_{r 22}\right)
\end{array}\right)
\end{aligned}
$$


is also invertible. Thus $e_{2}+\sum a_{r}^{*} a_{r}$ is invertible in $A_{2}$.

2.4. Proof of The Theorem. We have shown that the theorem holds for $n=$ $2^{1}$. If we assume the theorem for $n=2^{m}$, then it follows for $n=2^{(m+1)}$ from the fact that the *-algebra of $2^{(m+1)} \times 2^{(m+1)}$ matrices over $A$ is *-isomorphic to the *-algebra of $2 \times 2$ matrices over $A_{2^{n}}$ by partitioning. Thus the theorem holds for all $n=2^{m}$.

Now let $n$ be an arbitrary positive integer. Choose a positive integer $m$ such that $n<2^{m}$. Then $A_{n}$ is ${ }^{*}$-isomorphic to the ${ }^{*}$-subalgebra of all $2^{m} \times 2^{m}$ matrices over $A$ of the form

$$
\left(\begin{array}{cc}
a & 0 \\
0 & e_{\left(2^{m}-n\right)}
\end{array}\right), \quad \text { where } a \text { is in } A_{n} .
$$

The inverse of a matrix of this form, if it exists in $A_{2^{m}}$, is of the form

$$
\left(\begin{array}{cc}
a^{\prime} & 0 \\
0 & e_{\left(2^{m}-n\right)}
\end{array}\right), \quad \text { where } a^{\prime} \text { is in } A_{n} \text { and } a^{\prime} a=e_{n}=a a^{\prime} .
$$

Thus, if $A_{2^{m}}$ is $k$-symmetric, then also $A_{n}$ is $k$-symmetric. This proves the theorem for every positive integer $n$.

2.5. Example. The field $C\left(x_{1}, \ldots, x_{n}\right)$ of fractions of complex polynomials in $x_{1}, \ldots, x_{n}$, with involution defined by $x_{i}^{*}=x_{i}, i=1,2, \ldots, n$, is an example of a nonnormable ${ }^{*}$-algebra which is $k$-symmetric for every positive integer $k$.

3. Extensions. Let $A$ be a ${ }^{*}$-algebra and $I$ a ${ }^{*}$-ideal in $A$. Clearly, if $A$ is symmetric, then also $I$ and $A / I$ are symmetric. The converse is still an open problem (even for Banach *-algebras). Here are some observations concerning this problem.

3.1. Observe that $A$ is a ${ }^{*}$-ideal in $A_{e}$ such that the quotient algebra $A_{e} / A$,

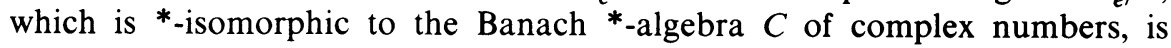
symmetric. Thus our Lemma 2.1 is a special case of this problem.

3.2. The radical $R$ of a ${ }^{*}$-algebra $A$ is a ${ }^{*}$-ideal in $A$ and every radical *algebra is symmetric. Furthermore, $A$ is symmetric if and only if $A / R$ is symmetric.

3.3. It is well known that a Banach *-algebra is symmetric if and only if it is Hermitian, i.e. every Hermitian element has real spectrum.

Now let $A$ be commutative and assume that $I$ and $A / I$ are Hermitian. We claim that then also $A$ is Hermitian. Indeed, if $h$ is a Hermitian element in $A$, then, since $A / I$ is Hermitian, $(e+i h) x=e+a+i b$, for some $x$ in $A$, and Hermitian $a$ and $b$ in $I$. Thus $(e+i h)(x-a)=e+i(b-h a)$, where $b-h a$ is a Hermitian element in $I$. Since $I$ is Hermitian, $e+i(b-h a)$ is invertible, and hence also $e+i h$. This shows that the spectrum of $h$ in $A$ is real.

3.4. A Banach ${ }^{*}$-algebra is called a $B^{*}$-algebra, if $\left\|a^{*} a\right\|=\|a\|^{2}$ for all elements $a$. It is well known that every $B^{*}$-algebra is symmetric.

Now assume that $I$ and $A / I$ are $B^{*}$-algebras. Then also $A$ is a $B^{*}$-algebra with respect to the norm

$$
\|a\|=\max \{\|a+I\|, \sup \{\|a x\|: x \text { in } I \text { and }\|x\| \leq 1\}\} .
$$


4. Completion. Let $A$ be a normed algebra with continuous involution. Then the completion $\tilde{A}$ of $A$ is a Banach *-algebra. We do not know if the completion $\tilde{A}$ is Hermitian whenever $A$ is Hermitian. Here are some remarks concerning this problem.

4.1. For any ${ }^{*}$-algebra $A$ consider the ${ }^{*}$-algebra $A_{0}$ of all infinite matrices $a=\left(a_{i j}\right)$ with $a_{i j}=0$ for almost all $i$ and $j$. If $A$ is $k$-symmetric for all $k=1,2,3, \ldots$, then $A_{0}$ has the same property.

In the special case where $A$ is a symmetric Banach algebra with continuous involution, the completion of $A_{0}$ with respect to the norm $\|a\|=\sum\left\|a_{i j}\right\|$ is again symmetric (H. Leptin, private communication).

4.2. In a commutative Banach algebra the spectrum is continuous. Thus, if $A$ is a commutative Hermitian normed algebra with continuous involution, then the completion of $A$ is again Hermitian.

4.3. The product $\prod A_{i}$ of a family of ${ }^{*}$-algebras $A_{i}$ is a *-algebra with respect to the involution $\left(a_{i}\right)^{*}=\left(a_{i}^{*}\right)$. The quasi-inverse of a quasi-regular element $\left(a_{i}\right)$ in $\prod A_{i}$ is given by $\left(-a_{i}\left(e-a_{i}\right)^{-1}\right.$ ) (with $\left\|a_{i}\left(e-a_{i}\right)^{-1}\right\|$ $\leq\left\|a_{i}\right\|\left(1-\left\|a_{i}\right\|\right)^{-1}$ if $A_{i}$ is normed and $\left.\left\|a_{i}\right\|<1\right)$. Hence the product $\prod A_{i}$ of a family of ${ }^{*}$-algebras $A_{i}$ is symmetric if and only if each $A_{i}$ is symmetric.

A similar remark holds for the ${ }^{*}$-ideal $\sum A_{i}$ of all $\left(a_{i}\right)$ in $\prod A_{i}$ with $a_{i}=0$ for almost all $i$, for the normed *-algebra of all $\left(a_{i}\right)$ in $\prod A_{i}$ with $\left\|\left(a_{i}\right)\right\|$ $=\sum\left\|a_{i}\right\|<\infty$, and for the normed * -algebra of all $\left(a_{i}\right)$ in $\prod A_{i}$ with $\left(\left\|a_{i}\right\|\right)$ vanishing at infinity and norm $\left\|\left(a_{i}\right)\right\|=\sup \left\|a_{i}\right\|$, when the ${ }^{*}$-algebras $A_{i}$ are normed.

\section{REFERENCES}

1. D. W. Bailey, On symmetry in certain group algebras, Pacific J. Math. 24 (1968), 413-419. MR 39 \#6085.

2. R. A. Bonic, Symmetry in group algebras of discrete groups, Pacific J. Math. 11 (1961), 73i94. MR 22 \#11281.

3. R. S. Doran, A generalization of a theorem of Civin and Yood on Banach *-algebras, Bull. London Math. Soc. 4 (1972), 25-26. MR 46 \#2442.

4. W. Glaser, Symmetrie von verallgemeinerten $L^{1}$-Algebren, Arch. Math. 20 (1969), 656-660. MR 41 \# 7448.

5. K. B. Laursen, Symmetry of generalized group algebras, Proc. Amer. Math. Soc. 25 (1970), 318-322; erratum, ibid. 42 (1974), 646; MR 41 \# 843.

6. H. Leptin, On symmetry of some Banach algebras, Pacific J. Math. 53 (1974), 203-206.

7. J. Wichmann, Hermitian *-algebras which are not symmetric, J. London Math. Soc. (2) 8 (1974), 109-112.

Department of Mathematics, TeXas Christian University, Fort Worth, Texas 76129

Current address: Department of Mathematics, Louisiana State University, Baton Rouge, Louisiana 70803 